Original Research Article

\title{
Multiple hepatocellular carcinoma: Long-term outcomes following resection beyond actual guidelines. An Italian multicentric retrospective study
}

\author{
Ilenia Bartolini ${ }^{\text {a, * }}$, Tommaso Nelli a , Nadia Russolillo ${ }^{b}$, Alessandro Cucchetti ${ }^{\text {, }}$ \\ Benedetta Pesi ${ }^{\mathrm{a}}$, Luca Moraldi $^{\mathrm{d}}$, Alessandro Ferrero ${ }^{\mathrm{b}}$, Giorgio Ercolani ${ }^{\mathrm{c}}$, Gianluca Grazi ${ }^{\mathrm{e}}$, \\ Giacomo Batignani ${ }^{\mathrm{a}}$ \\ a Department of Experimental and Clinical Medicine, AOU Careggi, Largo Brambilla 3, 50134, Florence, Italy \\ ${ }^{\mathrm{b}}$ Department of HPB and Digestive Surgery, Mauriziano Umberto I Hospital, Largo Filippo Turati 62, 10128, Turin, Italy

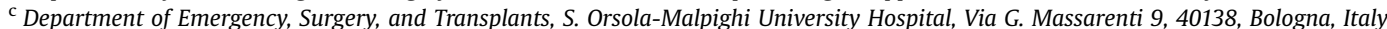 \\ d Surgical Oncology and Robotics, Department of Oncology, AOU Careggi, Largo Brambilla 3, 50134, Florence, Italy \\ e General and Hepatopancreatobiliary Surgery Unit, Regina Elena National Cancer Institute, Via E. Chianesi 53, 00128, Rome, Italy
}

\section{A R T I C L E I N F O}

\section{Article history:}

Received 9 November 2020

Received in revised form

6 January 2021

Accepted 16 January 2021

\section{Keywords:}

Multiple HCC

Liver resection

Liver surgery indication

Guidelines

Prognostic factors

\begin{abstract}
A B S T R A C T
Background: Hepatocellular carcinoma (HCC) is frequently diagnosed as multinodular. This study aims to assess prognostic factors for survival and identify patients with multiple HCC who may benefit from surgery beyond the Barcelona Clinic Liver Cancer classification indications.

Methods: This retrospective study included all the consecutive patients from 4 Italian tertiary centers receiving liver resection for naive multiple HCC between 1990 and 2012 to have a potential follow-up of 5 years.

Results: Included patients were 144 . Ninety-day morbidity and mortality rates were $38.3 \%$ and $8.3 \%$, respectively. The 5 -year overall and disease-free survival rates were $33.3 \%$ and $19.1 \%$, respectively. Tumor size $<3 \mathrm{~cm}$, bilirubin, Child-Pugh A, BCLC-A stage, being within "up-to-7" criteria, and minor resections resulted in prognostic factors. The Child-Pugh score resulted in an independent prognostic factor. Conclusions: Surgery may be related to good outcomes in selected patients with multiple HCC.
\end{abstract}

() 2021 Elsevier Inc. All rights reserved.

\section{Introduction}

Hepatocellular carcinoma (HCC) is the most frequent primitive liver tumor ${ }^{1}$ and it is a highly lethal neoplasm being the third leading cause of cancer mortality worldwide. ${ }^{2}$ Despite the introduction and implementation of structured follow-up programs, HCC is still diagnosed in advanced/multiple form in approximately $44 \%$ of cases. $^{3}$ Differentiation between the multifocal and multinodular form is usually tricky even though these two types may have different prognosis. ${ }^{4}$

Over the past 20 years, several guidelines for the management of HCC have been proposed. The Barcelona Clinic Liver Cancer

\footnotetext{
* Corresponding author. Hepatobiliary Surgery Unit, Department of Clinical and Experimental Medicine, University of Florence, AOU Careggi, Largo Brambilla 3, 50134, Florence, Italy.

E-mail address: ilenia.bartolini@gmail.com (I. Bartolini).
}

(BCLC) classification is a prognostic and staging system that considers tumor number and dimension, liver function, and patient conditions and indicates tumor treatment. The BCLC is a widely used classification and it is endorsed by the American Association for the Study of Liver Disease (AASLD) and the European Association for the Study of Liver (EASL). ${ }^{1}$ According to this guideline, only $30-40 \%$ of the patients with HCC are suitable for curative treatments. ${ }^{5}$ In the presence of multiple nodules, when specific criteria are met, liver transplantation (LT) is the best option removing both the tumor and the failing liver in which future malignancies may regrow. However, the organ shortage limits the availability of LT with long waiting lists and significant dropout rates.

Consequently, the need for alternative treatments, improvements in surgical technique, and ameliorations in perioperative care $^{6}$ have driven further surgery indications. Many papers demonstrated that hepatic resections might provide good short outcomes and improve survival in well-selected patients with 
multiple HCC. ${ }^{7-10}$ Furthermore, neither portal hypertension (PHT) or large nodules are reported to be an absolute contraindication to surgery. ${ }^{11-14}$ In Eastern countries, new evidence-based flow-charts with broader indications for resective surgery have already been reported. ${ }^{15,16}$

This Italian retrospective multicentric study aimed to find prognostic factors for survival in patients with multiple HCC to identify which of them could benefit from surgical resection beyond actual guidelines.

\section{Materials and methods}

\section{Study design and patients}

This multicentric, retrospective study involved four Italian referral centers: Mauriziano Hospital, Turin; Regina Elena National Cancer Institute, Rome; Sant'Orsola-Malpighi Hospital, Bologna, and Careggi University Hospital, Florence (coordinator center).

The centers were asked to fill a database including all the consecutive patients who underwent surgery for naive multiple HCC from January 1990 to May 2012. Since the differentiation between multifocal and multinodular forms is challenging, all the patients were considered as suffering from "multiple" HCC.

Inclusion criteria were radiological diagnosis of multiple HCCs; no radiologic macroscopic vascular invasion; Child-Pugh class A or B7; absence of ascites and no signs of PHT (defined as the presence of esophageal varices or a platelet count of $<60000 / \mathrm{mL}$ in association with splenomegaly). The exclusion criterion was a previous treatment (transarterial chemoembolization -TACE-, liver resections, or ablation treatment).

\section{Perioperative evaluation}

Several demographic and preoperative clinical variables, including liver status, were analyzed. Preoperative diagnostic workup included a Computed Tomography (CT) scan and Magnetic Resonance Imaging (MRI), as best indicated for each patient. Tumor size was defined as the major radiological diameter of the largest tumor. Patients were stratified according to the Child-Pugh score, BCLC stage, ${ }^{5}$ "up-to- 7 " criteria, ${ }^{17}$ and ALBI score. ${ }^{18}$ Patients within "up-to-7" criteria were those with 7 as the sum of the major diameter in centimeter of the largest tumor with the tumor's total number. The ALBI score was calculated with the formula $\mathrm{x}=(\log 10$ bilirubin $\times 0.66)+($ albumin $\times-0.085)$ and patients were classified as ALBI grade 1 if $\mathrm{x} \leq-2.60$ or ALBI grade 2 if $-2.60<\mathrm{x} \leq-1.39$.

According to Brisbane's classification, the type of resection was divided into major resection (removal of at least 3 segments) and minor resection. Minor resections comprehended anatomic resections and nonanatomic (parenchymal sparing) resections. Intraoperative ultrasonography (IOUS) was routinely used to complete the staging and to confirm preoperative planning.

\section{Postoperative management and patient follow-up}

Postoperative morbidity was evaluated according to ClavienDindo classification. ${ }^{19}$

Patients were followed every 3, 6, and 12 months for the first year after surgery and then every six months or annually with the US or a CT scan according to patient and tumor characteristics, for 5 years after surgery. Overall survival (OS) was defined as the period between surgery and the last available follow-up or death. Diseasefree survival (DFS) was defined as the period between surgery and the first recurrence diagnosis. Recurrence of HCC was defined as a new tumor diagnosis.

Every decision about patient treatment was taken after a multidisciplinary evaluation.

\section{Statistical analysis}

Continuous variables were expressed as mean \pm standard deviation (SD). Univariate analysis was performed using $t$-test or chisquare test, as appropriate. Survival rates were obtained using Kaplan-Meier curves and compared using the log-rank test. The relation between the clinical variables and OS/DFS after resection was evaluated by the univariate analysis. Variables significantly associated with survival in univariate analysis were tested with the Cox multivariate regression model. Statistical significance was defined as a p value $<0.05$.

All collected information was analyzed using the SPSS for Windows 21.0 software package (SPSS Inc., Chicago, Illinois, USA).

\section{Results}

\section{Patient characteristics}

A total of 144 patients was included in the study.

Preoperative characteristics and baseline demographics are summarized in Table 1. Hepatitis was mostly related to virus infection and coinfection of hepatitis $B$ virus and hepatitis $C$ virus

Table 1

Demographics and clinical characteristics of the Patients.

\begin{tabular}{|c|c|c|}
\hline $\begin{array}{l}\text { Patients' } \\
\text { characteristics }\end{array}$ & & \\
\hline Age (y) & & $66 ., 3 \pm 10 ., 5$ \\
\hline Sex & & \\
\hline & Male & $114(79.2 \%)$ \\
\hline & Female & $30(20.8 \%)$ \\
\hline Hepatitis Etiolo & & \\
\hline & Viral hepatitis & $93(64.6 \%)$ \\
\hline & $\mathrm{HCV}$ & 77 (53.5\%) \\
\hline & $H B V$ & $18(12.5 \%)$ \\
\hline & Alcoholic & $36(25 \%)$ \\
\hline & Idiopathic & $23(16 \%)$ \\
\hline Cirrhosis (N, \%) & & \\
\hline & No & 35 (24.3\%) \\
\hline & Yes & $109(75.7 \%)$ \\
\hline & Viral Cirrhosis & $82(75.2 \%)$ \\
\hline & Alcoholic Cirrhosis & 21 (19.3\%) \\
\hline & Idiopathic Cirrhosis & $6(5.5 \%)$ \\
\hline Liver Function & & \\
\hline Blood tests & & \\
\hline & $\mathrm{GPT}(m U / m l)$ & $77.03 \pm 74.5$ \\
\hline & GOT $(m U / m l)$ & $77.95 \pm 77.3$ \\
\hline & INR & $1.08 \pm 0.14$ \\
\hline & Albumin $(g / d L)$ & $3.82 \pm 0.52$ \\
\hline & Bilirubin $(m g / d l)$ & $0.94 \pm 0.61$ \\
\hline & Platelets $\left(10^{3} / \mu l\right)$ & $171.07 \pm 108.3$ \\
\hline Child-Pugh scor & & \\
\hline & A & $131(91 \%)$ \\
\hline & B & $13(9 \%)$ \\
\hline BCLC stage $(\mathrm{N}$, & & \\
\hline & A & $40(27.8 \%)$ \\
\hline & B & $104(72.2 \%)$ \\
\hline "Up-to-7" criter & & \\
\hline & In & $86(59.7 \%)$ \\
\hline & Out & $58(40.3 \%)$ \\
\hline ALBI score & & \\
\hline & $x \leq-2.6$ & $46(38.6 \%)$ \\
\hline & $-2.6<x \leq-1.39$ & $73(61.4 \%)$ \\
\hline
\end{tabular}

$\mathrm{HCV}=$ Hepatitis C Virus

$\mathrm{HBV}=$ Hepatitis B Virus

GPT $=$ Glutamic Pyruvic Transaminase.

GOT $=$ Glutamic Oxaloacetic Transaminase.

$\mathrm{INR}=$ International Normalised Ratio.

$\mathrm{BCLC}=$ Barcelona Clinic Liver Cancer. 
Table 2

Tumor characteristics.

\begin{tabular}{lll}
\hline Tumor characteristics & & \\
\hline Nodules (N, \%) & $2-3$ & $118(81.9 \%)$ \\
& $4+$ & $26(18.1 \%)$ \\
Diameters (N,\%) & $4.5 \pm 3.7($ mean \pm SD) & \\
& $<3 \mathrm{~cm}$ & $51(35.4 \%)$ \\
& $3-5 \mathrm{~cm}$ & $50(34.7 \%)$ \\
& $>5 \mathrm{~cm}$ & $43(29.9 \%)$ \\
\hline
\end{tabular}

$\mathrm{SD}=$ Standard Deviation

was discovered in 2 patients. Eight patients had multifactorial hepatitis. A great majority of the patients had liver cirrhosis. Most of the cirrhotic patients were classified as Child-Pugh A and BCLC-B, according to BCLC classification.

Tumor characteristics are summarized in Table 2, while the frequency of the different hepatic resections performed is outlined in Table 3. Minor resections were the most frequently performed operations.

\section{Postoperative short-term outcomes and survival analysis}

Morbidity rate was $38.3 \%$. Thirty- and 90-day mortality rates were $7 \%$ and $8.3 \%$, respectively. Considering separately the mortality rate in the periods 1990-1999 and 2000-2012, 30-day mortality rates were $22.6 \%$ and $2.5 \%$, respectively, while 90 -day mortality rates were $22.6 \%$ and $4.4 \%$, respectively.

The 1-, 3-, and 5-year OS rates were $80.6 \%, 43.8 \%$ and $33.3 \%$, respectively (Fig. 1a) while the 1-, 3-, and 5-year DFS rates were $65.5 \%, 35 \%$ and 19.1\%, respectively (Fig. 1b). Clinicopathologic

\section{factors affecting survival in resected multiple HCC.}

Several clinicopathologic factors were analyzed in the univariate analysis to detect prognostic factors for survival following surgical resection (Table 4).

Sex was not a prognostic factor even in the presence of a slightly better 3- and 5-years OS in male patients (Fig. 2). The presence of cirrhosis was not found to be a significant prognostic factor.

The major radiological diameter of the largest tumor resulted in a significant prognostic factor for OS $(\mathrm{p}=0.024)$ : patients with a smaller than $3 \mathrm{~cm}$ tumor had the best prognosis (Fig. 3a). On the contrary, tumor number was not a significant prognostic factor (Fig. 3b).

Non-cirrhotic and Child A patients had a significantly better prognosis when compared to Child B patients. All Child B patients died within 30 months from surgery (Fig. 4a). BCLC classification was identified as a significant prognostic factor, with patients within stage A having the best prognosis (Fig. 4b). Stratification, according to the ALBI score, did not result in a prognostic factor $(\mathrm{p}=0.547)$.

Patients within "up-to-7" criteria had a prognostic advantage when compared with patients outside "up-to-7" criteria $(\mathrm{p}=0.023)$ (Fig. 5).
Minor resections allowed a better prognosis compared to major resections with a marginally significant difference $(\mathrm{p}=0.054)$ (Fig. 6a), while there were no statistical differences in DFS between patients undergoing major or minor (typical/atypical) resections (Fig. 6b).

Tumor size, total bilirubin, Child-Pugh score, BCLC stage, "up-to7" criteria in or out, and type of resection were tested with multivariate Cox analysis. Only the Child-Pugh score resulted in an independent prognostic factor for survival (Table 5).

\section{Discussion}

Despite follow-up programs, HCC is still diagnosed in a multiple presentation in a considerable percentage of the patients. ${ }^{20}$ On a theoretical basis, LT offers the best results, even beyond Milan criteria. ${ }^{17}$ However, the organ shortage remains an overwhelming and unsolved problem. In the past years, multiple presentation of HCC was considered an unfavorable prognostic factor being associated with early recurrence and a high probability of postoperative complications when resection was performed. ${ }^{5}$ Progressive technological and technical improvements (including greater use of IOUS and better surgical coagulation devices), growing adoption of minimally invasive surgery and parenchymal-sparing techniques along with better perioperative management, led to the achievement of superior postoperative outcomes. Furthermore, a higher probability of oncological radicality and a lower impact on patients and liver function ${ }^{21}$ may be obtained, as confirmed in our paper. Considering separately the periods 1990-1999 and 2000-2012, the mortality rate changes radically from $22.6 \%$ to $2.5 \%$, below the recommended $3 \%$ proposed by the recently published EASL guidelines. ${ }^{1}$ However, the discrepancy between these two periods may also be related to the different sample sizes (31 patients treated before 2000 versus 113 patients treated after 2000). The 1-, 3 -, and 5-year OS were $80.6 \%, 43.8 \%$, and $33.3 \%$, respectively, while the same rates for the BCLC-B patients (72.2\% of the group) were $74 \%, 37.5 \%$, and $26.9 \%$, respectively. According to the BCLC guideline, TACE should be indicated for BCLC-B patients providing an expected OS of about 30 months ${ }^{1,5}$ with a lower morbidity rate when compared with surgery. ${ }^{14,22-24}$ In a large meta-analysis of Yang, ${ }^{25}$ including 9580 patients with single large or multiple HCC, 1-, 3and 5-years OS rates after TACE were 68.3\%, 31.5\%, and 17.5\%.

Safety and feasibility of hepatic resection associated with good survival outcomes in well-selected patients with multiple HCC have been previously reported. ${ }^{7,26-29}$ Torzilli et al. ${ }^{9}$ published the socalled "East-West Study", including results from 2046 patients from 9 different referral centers from America, Asia, and Europe. The 90 -day postoperative mortality was $2.7 \%$ despite BCLC class and 5-years OS and DFS rates were $57 \%$ and $27 \%$, respectively, for BCLC-B patients. In another recent systematic review of large/ multiple HCC performed by Zhong et al. ${ }^{29}$ involving 14808 patients, the global 5-year OS and DFS were $42 \%$ and $26 \%$, respectively; intrahospital mortality rate ranged from $2.7 \%$ to $7.3 \%$ and morbidity rate ranged from $26.6 \%$ to $32.3 \%$. However, these papers

Table 3

Type of resection performed.

\begin{tabular}{|c|c|c|c|c|}
\hline & & BCLC* A & BCLC* B & \\
\hline Major Resections & $40(27.8 \%)$ & $3(7.5 \%)$ & 37 (92.5\%) & 7.5\% vs $92.5 \%$ \\
\hline Minor Resections & $104(72.2 \%)$ & 37 (35.6\%) & $67(64.4 \%)$ & $35.6 \%$ vs $64.4 \%$ \\
\hline typical & $50(48.1 \%)$ & 13 (35.1\%) & $37(55.2 \%)$ & \\
\hline \multirow[t]{2}{*}{ atypical } & $54(51.9 \%)$ & 24 (64.9\%) & $30(44.8 \%)$ & \\
\hline & & $7.5 \%$ vs $92.5 \%$ & $35.6 \%$ vs $64.4 \%$ & \\
\hline
\end{tabular}

$\mathrm{BCLC}=$ Barcelona Clinic Liver Cancer Classification. 


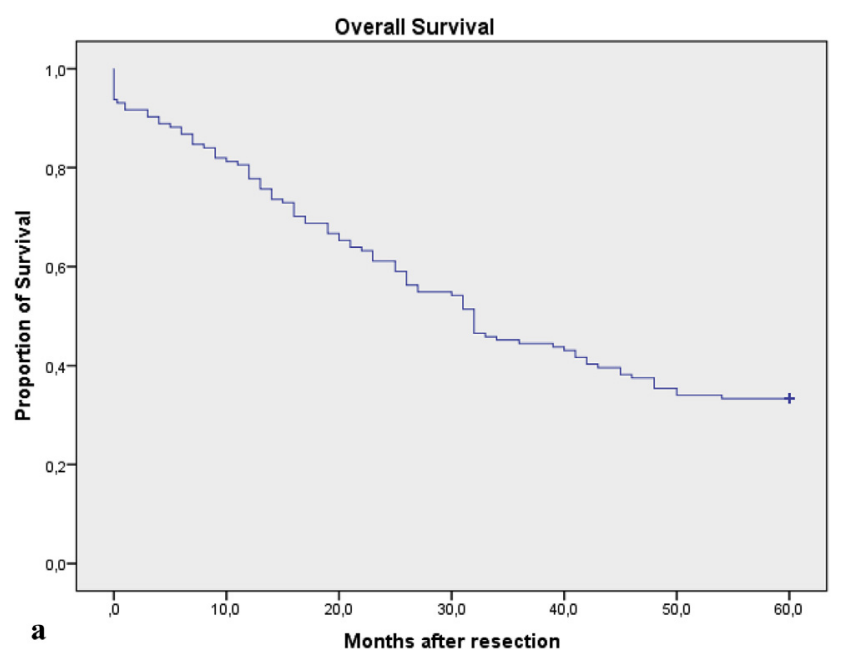

Fig. 1a. Survival analysis. a. Kaplan-Meier curve of Overall Survival.

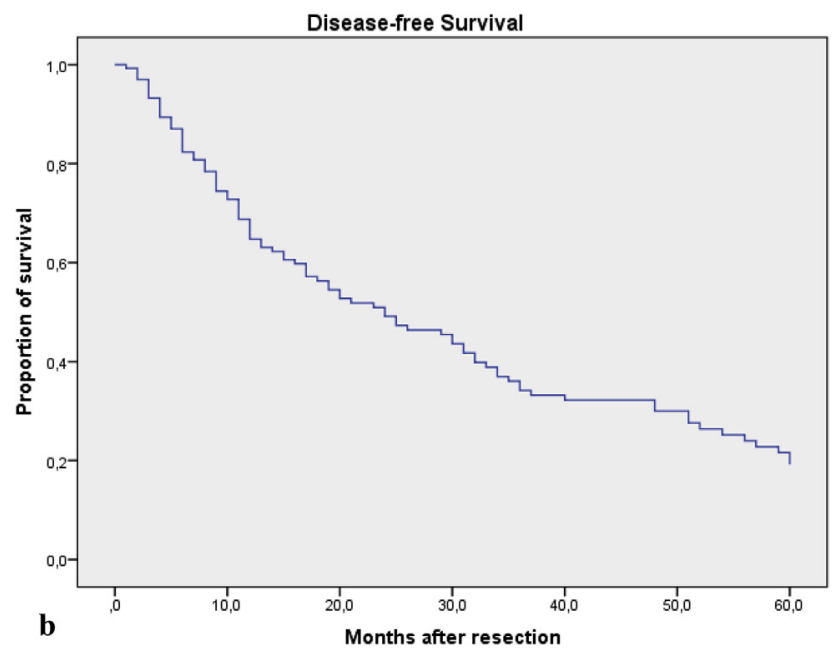

Fig. 1b. Kaplan-Meier curve of Disease-Free Survival.

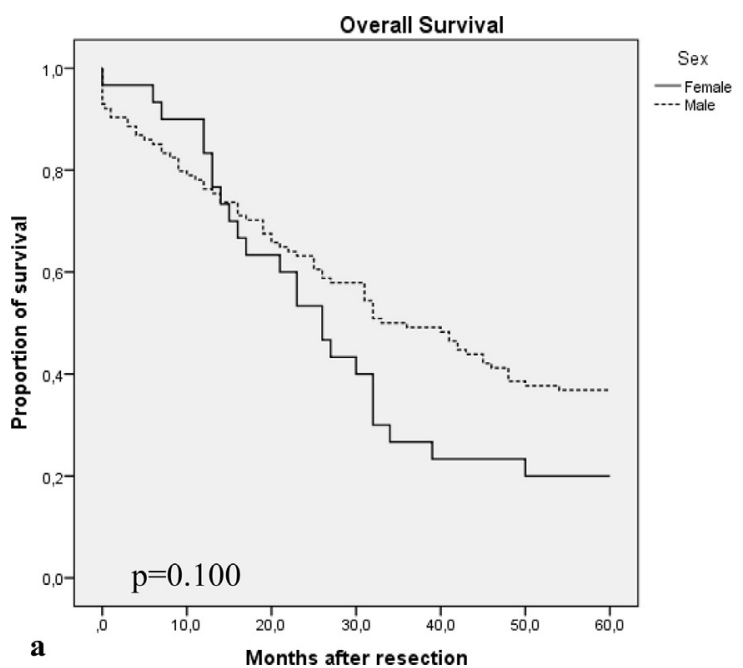

Table 4

Univariate Analysis of prognostic factors for Overall Survival.

\begin{tabular}{|c|c|c|c|}
\hline Variable & $\mathrm{N}(\%)$ & 5-years survival (\%) & $\mathrm{p}$ value \\
\hline \multicolumn{3}{|l|}{ Bilirubin } & $<0.001$ \\
\hline \multicolumn{3}{|l|}{ Tumor size } & 0.024 \\
\hline \multicolumn{3}{|l|}{ Child-Pugh } & $<0.001$ \\
\hline$A$ & 131 (91\%) & $36.6 \%$ & \\
\hline$B$ & 13 (9\%) & $0 \%$ & \\
\hline \multicolumn{3}{|l|}{ BCLC } & 0.010 \\
\hline$A$ & $40(27.8 \%)$ & $50 \%$ & \\
\hline$B$ & $104(72.2 \%)$ & $26.9 \%$ & \\
\hline \multicolumn{3}{|c|}{ Major vs. Minor Resection } & 0.054 \\
\hline Major & $40(27.8 \%)$ & $22.5 \%$ & \\
\hline Minor & $104(72.2 \%)$ & $37.5 \%$ & \\
\hline \multicolumn{3}{|c|}{ "Up-to-7" criteria } & 0.023 \\
\hline In & $86(59.7 \%)$ & $39.5 \%$ & \\
\hline Out & $58(40.3 \%)$ & $24.1 \%$ & \\
\hline \multicolumn{3}{|l|}{ ALBI score } & 0.33 \\
\hline
\end{tabular}

BCLC $=$ Barcelona Clinic Liver Cancer Classification.

and several others ${ }^{20,29,30}$ considered the whole BCLC intermediate stage that comprehends a heterogeneous group of patients. In particular, it is recognized that large solitary HCCs have a better prognosis than multiple HCC. ${ }^{7}$

Indeed, the patient's selection is of paramount importance to achieve good results, especially when pushing indication beyond existing guidelines.

In this paper, univariate analyses revealed that tumor size smaller than $3 \mathrm{~cm}$, bilirubin level, absence of cirrhosis and Child A stage, BCLC-A, and being within Milan revised "up-to seven" criteria were significant prognostic factors associated with better outcomes.

Cirrhosis was not a prognostic factor in the present study, probably because of the small number of non-cirrhotic patients and the accurate selection of cirrhotic patients indicated for surgery.

As confirmed in our paper, tumor size is a well-known prognostic factor associated with the probability of vascular invasion and metastasization when bigger than $5 \mathrm{~cm} ., 9$

Tumor number was not a significant prognostic factor in the present paper. However, the patient group with 4-plus nodules was much smaller than the other (26 versus 118 ). This fact, associated with a good selection of the patients, may explain these results. Interestingly, stage A of BCLC classification (that includes tumor

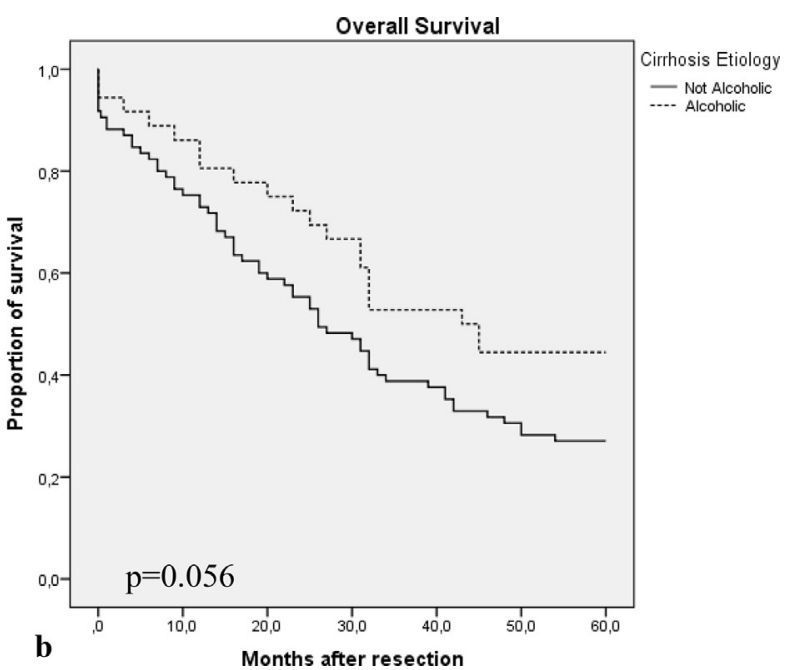

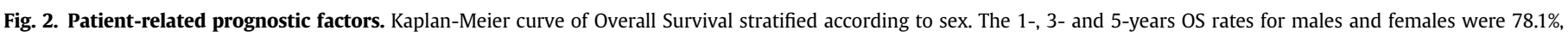
$49.1 \%$, and $36.8 \%$ versus $83.3 \%, 26.7 \%$, and $20 \%$, respectively, $\mathrm{p}=0.100$. 


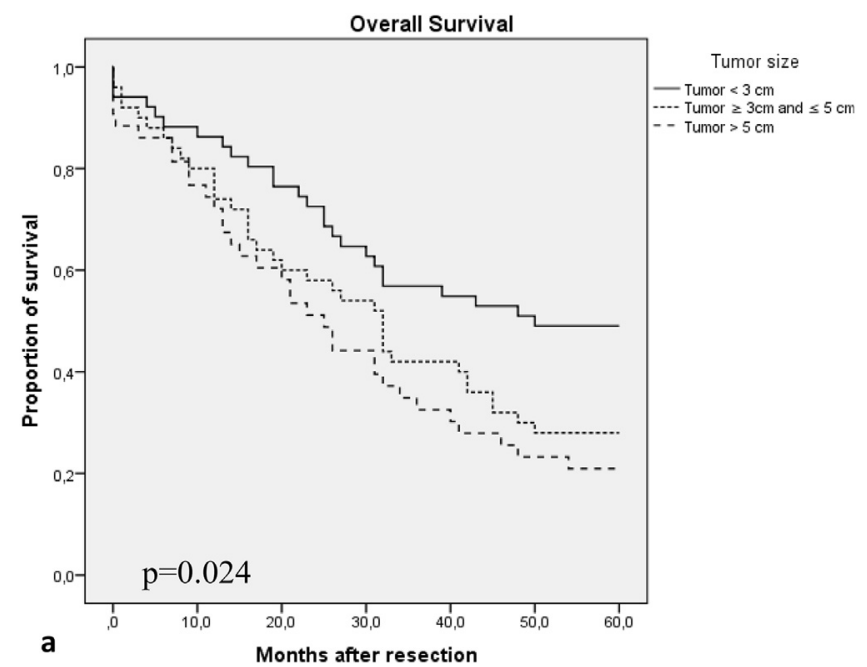

Fig. 3a. Tumor-related prognostic factors. a. Kaplan-Meier curve of Overall Survival stratified according to tumor size. Patients with a tumor smaller than $3 \mathrm{~cm}$ have a significant $(\mathrm{p}=0.024)$ better prognosis compared to the others with a 5-years OS rate of $49 \%$, while patients with a tumor size between 3 and $5 \mathrm{~cm}$ had a 5 -years OS rate of $20.9 \%$ and patients with a tumor size bigger than $5 \mathrm{~cm}$ had a 5 -years OS rate of $28 \%$.

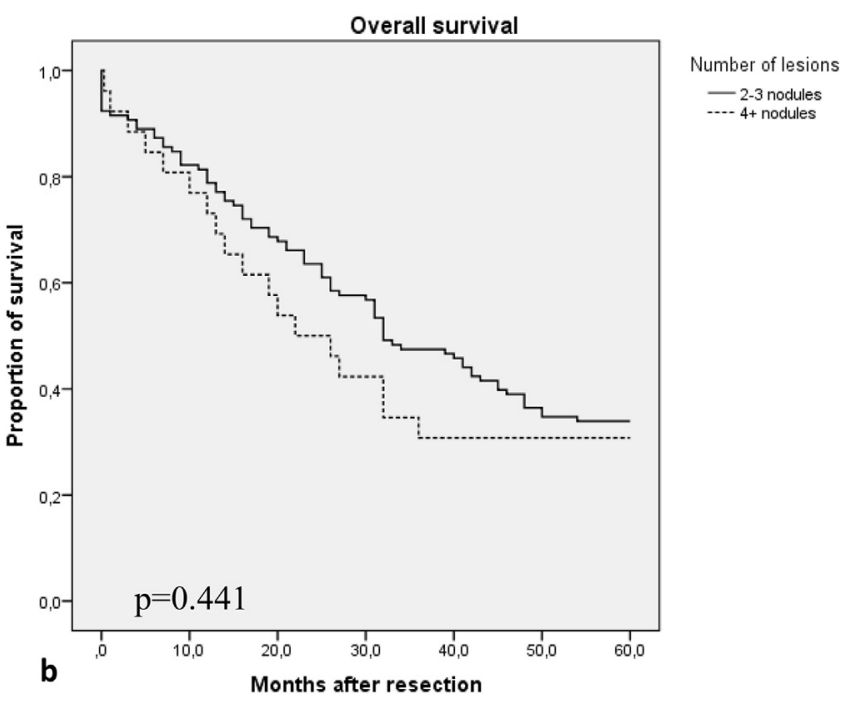

Fig. 3b. Kaplan-Meier curve of Overall Survival stratified according to tumor number. The 1 - 3 - and 5 -years OS rates were $81 \%, 46.6 \%$, and $33.9 \%$ versus $76.9 \%, 30.8 \%$, and $30.8 \%$ for patients with less than 3 nodules or at least 4 nodules, respectively. There is no statistically significant difference between the two groups $(\mathrm{p}=0.441)$.

diameter and number and liver function) and being within Milan "up-to-7" criteria resulted in a significantly better prognosis. Wada et al. $^{8}$ performed a recent retrospective analysis of 85 Child A patients with multiple HCC included in BCLC-A or BCLC-B stages, dividing them into three groups according to tumor size and number. The 5-years OS rates ranged from $37.1 \%$ to $75.2 \%$, and tumor size and number resulted in independent prognostic factors.

In the present study, the comparison between Child $A$ and Child $B$ patients showed a significantly worse prognosis for the latter. Child B subgroup was scarcely represented (13 patients, 9\%); however, none of those patients survived beyond 30 months from surgery.

Although the early and intermediate stages of the BCLC classification formally include Child A and Child B classes, ${ }^{5}$ most of the previously published papers about this topic analyzed only Child A

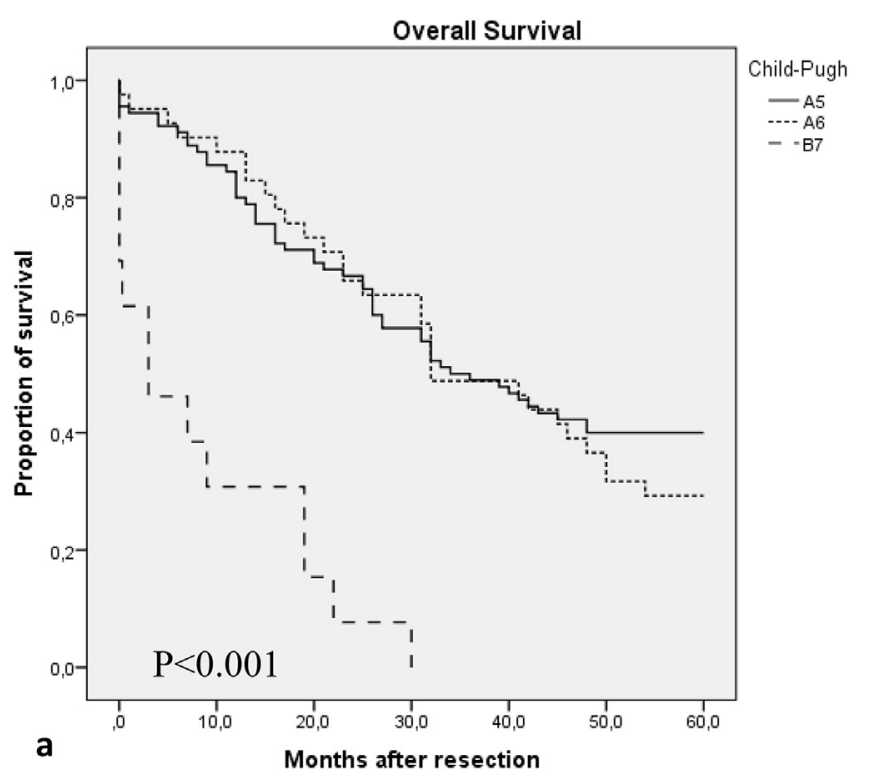

Fig. 4a. Overall survival stratified according to the Child-Pugh score and BCLC classification. a. Kaplan-Meier curve of Overall Survival stratified according to the ChildPugh score. Non-cirrhotic patients and Child A patients had a significantly ( $p<0.001$ ) better prognosis than Child B patients.

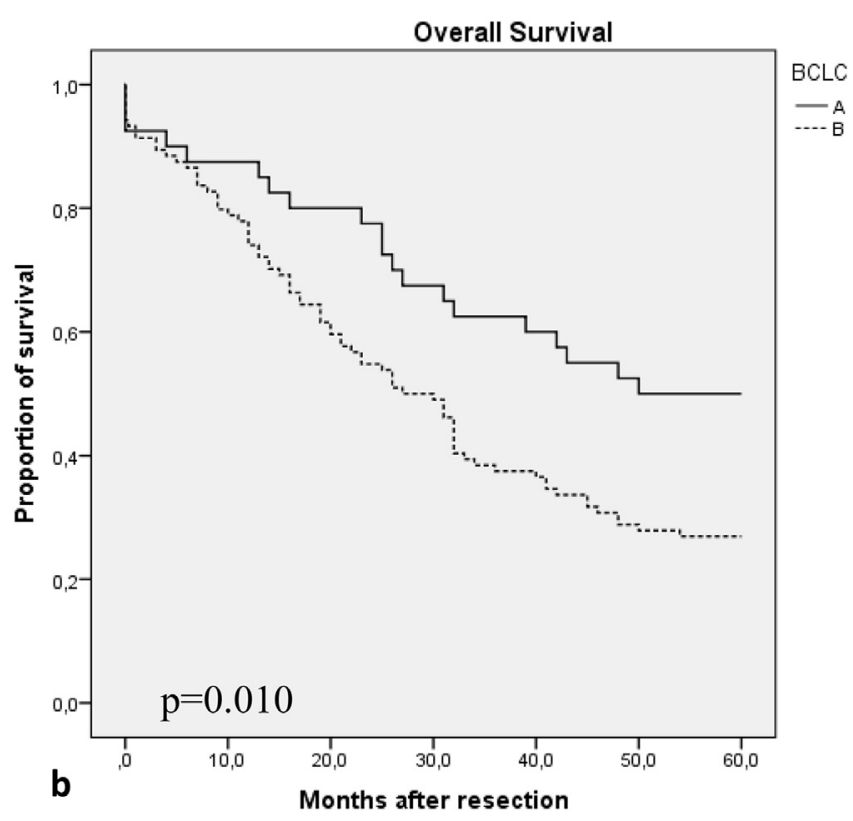

Fig. 4b. Kaplan-Meier curve of Overall Survival stratified according to BCLC stage classification. BCLC-A patients with multiple HCC had a 1-, 3-, 5-years overall survival rate of $87.5 \%, 62.5 \%$, and $50 \%$, respectively, compared to $77.9 \%, 37.5 \%$, and $26.9 \%$, respectively, for BCLC-B patients $(\mathrm{p}=0.010)$.

patients $^{8,14}$ or involved Child B patients in minimal percentages without performing Child class subgroup analysis. ${ }^{9,25}$ Consequently, Child B patients with multiple tumors presentation should probably receive treatments different from surgery, and only referral centers could evaluate resection in very selected Child B patients. However, definite conclusions cannot be drawn, and further studies may be needed to address this specific issue.

The high recurrence rate is another well-known problem related to multiple HCC. Parenchymal-sparing resections may provide survival benefit because of the lower impact on postoperative liver 


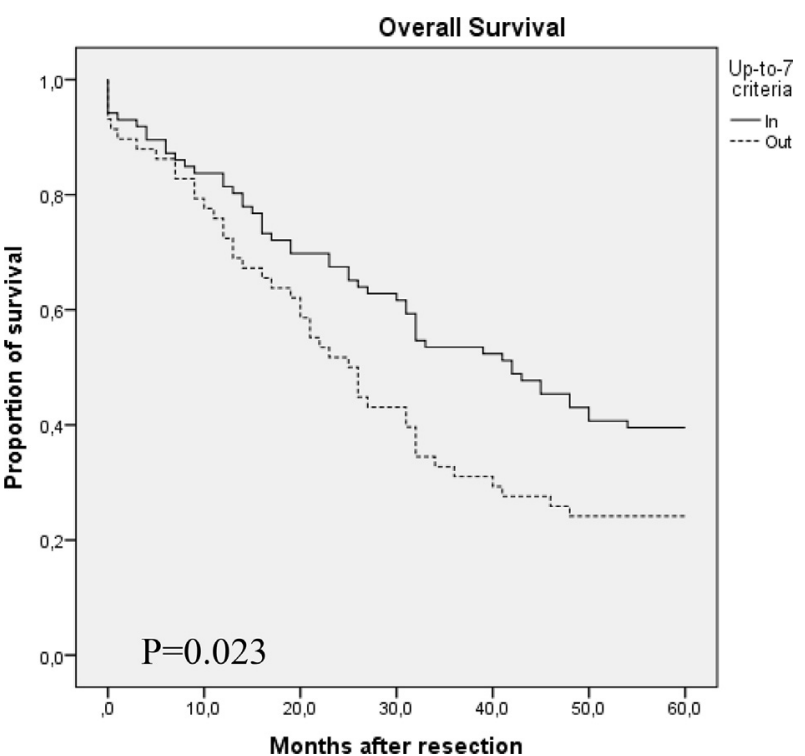

Fig. 5. Kaplan-Meier curve of Overall Survival stratified according to "up-to-7" criteria. Patients considerable within "up-to-7" criteria had a significantly $(\mathrm{p}=0.023)$ better prognosis with a 5-years overall survival rate of $39.5 \%$ compared to patients outside these criteria with a 5 -years overall survival rate of $24.1 \%$.

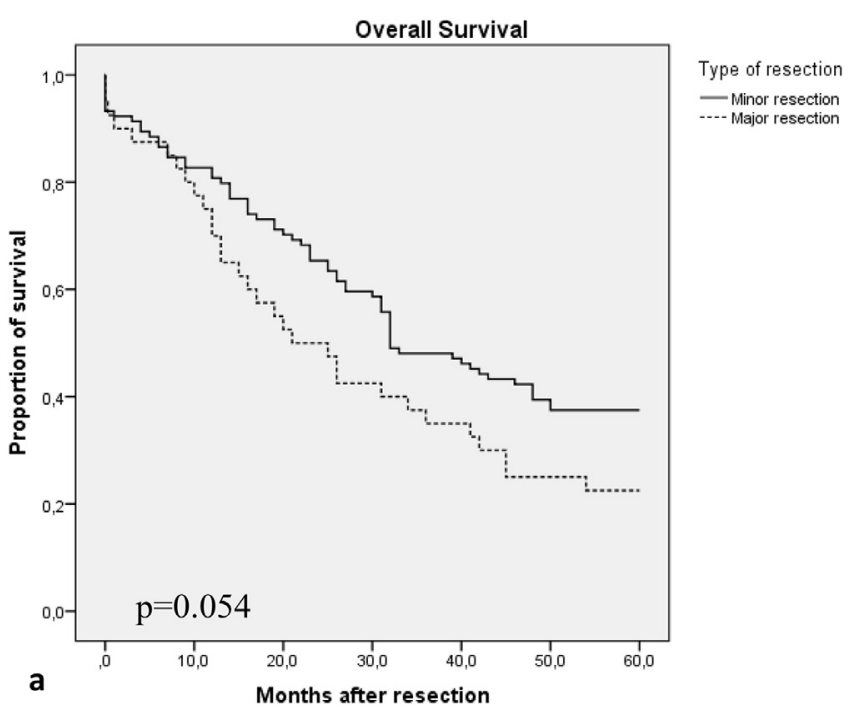

Fig. 6a. Kaplan-Meier curve of Overall Survival stratified according to types of resection performed. Minor resections were related to a marginally significant $(p=0.054)$ better prognosis when compared to major resections.

function and the greater possibility of performing repeated resection or ablation in case of recurrence. In the present paper, no statistically significant differences were found in DFS between major and minor resections, demonstrating that a more conservative approach was not less radical. Interestingly, almost all major hepatectomies are performed for BCLC-B patients.

This study has some drawbacks. It is a retrospective series with the consequent intrinsic selection bias. Although the group of patients analyzed is homogeneous, including only multifocal/multinodular HCC and with an extended follow-up, it is relatively small. The period's width may produce bias, such as the different mortality rates found dividing the analysis into two periods. There was not a control group to compare the results. Finally, different

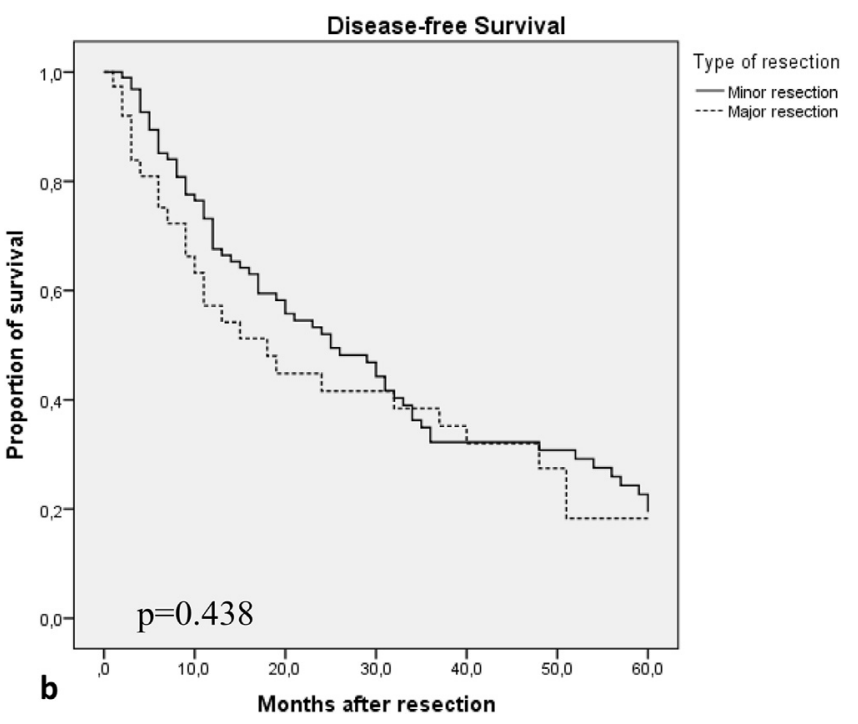

Fig. 6b. Kaplan-Meier curve of Disease-Free Survival stratified according to types of resection performed. DFS is not significantly different between the two groups $(\mathrm{p}=0.438)$

Table 5

Multivariate Analysis of prognostic factors for Overall Survival.

\begin{tabular}{llll}
\hline Variable & HR & $95 \% \mathrm{CI}$ & $\mathrm{p}$ value \\
\hline Bilirubin & 0.45 & $-0.164 /-1.041$ & 0.16 \\
BCLC & -0.259 & $-0.862 /-0.442$ & 0.42 \\
Child-Pugh score & -1.495 & $-2.523 /-0.765$ & $\mathbf{0 . 0 0 2}$ \\
"Up-to-7" & -0.194 & $-0.791 / 0.44$ & 0.50 \\
Tumor size & 0.004 & $-0.004 / 0.18$ & 0.43 \\
\hline
\end{tabular}

HR = Hazard Ratio.

$\mathrm{CI}=$ Confidential Interval.

$\mathrm{BCLC}=$ Barcelona Clinic Liver Cancer Classification.

treatment of patients who experienced recurrence may further influence survival results.

\section{Conclusion}

In conclusion, even in multiple HCC, hepatic resection may be considered a valid option providing good long-term outcomes with acceptable morbidity and mortality rates. Accurate choice of the patients with a multidisciplinary team and tailored treatment is of capital importance. Patients with multiple HCC should be evaluated for a surgical resection instead of palliative treatments, mostly when classified as Child A, as BCLC-A, when having a maximum tumor diameter lesser than $3 \mathrm{~cm}$, and when being within "up-to-7" criteria. Minor resections should be performed whenever possible.

On the contrary, Child B patients with multiple tumors presentation should not be resected, though definite conclusions cannot be drawn since they were scarcely represented in the present study. Similarly, considering the small number of patients with 4 or more lesions analyzed, these conclusions should be related to patients with 2 or 3 tumors.

However, in this light, the BCLC classification seems to be quite restrictive and the selection criteria for resection in the subgroup of multiple HCC need further evaluations.

\section{Funding}

This research did not receive any specific grant from funding agencies in the public, commercial, or not-for-profit sectors. 


\section{Data statement}

The dataset generated and analyzed during the current study is available from the corresponding author on reasonable request. Due to privacy reasons, the dataset will be provided in an anonymous format.

\section{Declaration of competing interest}

The authors declare that they have no financial relationship or any conflict of interests and they have nothing to disclose.

All authors acknowledge absence of conflict of interest of their co-authors, to the best of their knowledge.

\section{References}

1. Galle PR, Forner A, Llovet JM, et al. EASL clinical practice guidelines: management of hepatocellular carcinoma. J Hepatol. 2018;69(1):182-236. https:// doi.org/10.1016/j.jhep.2018.03.019.

2. Lafaro KJ, Demirjian AN, Pawlik TM. Epidemiology of hepatocellular carcinoma. Surg Oncol Clin. 2015;24(1):1-17. https://doi.org/10.1016/j.soc.2014.09.001.

3. Guadagnino V, Stroffolini T, Rapicetta M, et al. Prevalence, risk factors, and genotype distribution of hepatitis $C$ virus infection in the general population: a community-based survey in southern Italy. Hepatology. 1997;26(4): 1006-1011. https://doi.org/10.1002/hep.510260431.

4. Feo F, Pascale RM. Multifocal hepatocellular carcinoma: intrahepatic metastasis or multicentric carcinogenesis? Ann Transl Med. 2015;3(1). https://doi.org/ 10.3978/j.issn.2305-5839.2014.12.08

5. Llovet JM, Brú C, Bruix J. Prognosis of hepatocellular carcinoma: the BCLC staging classification. Semin Liver Dis. 1999;19(3):329-337. https://doi.org/ 10.1055/s-2007-1007122.

6. Wu CC, Cheng SB, Ho WM, Chen JT, Liu TJ, P'eng FK. Liver resection for hepatocellular carcinoma in patients with cirrhosis. Br J Surg. 2005;92(3):348-355. https://doi.org/10.1002/bjs.4838.

7. Kamiyama T, Orimo T, Wakayama K, et al. Survival outcomes of hepatectomy for stage B Hepatocellular carcinoma in the BCLC classification. World J Surg Oncol. 2017;15(1). https://doi.org/10.1186/s12957-017-1229-X.

8. Wada H, Eguchi H, Noda T, et al. Selection criteria for hepatic resection in intermediate-stage (BCLC stage B) multiple hepatocellular carcinoma. Surgery. 2016;160(5):1227-1235. https://doi.org/10.1016/j.surg.2016.05.023.

9. Torzilli G, Belghiti J, Kokudo N, et al. A snapshot of the effective indications and results of surgery for hepatocellular carcinoma in tertiary referral centers: is it adherent to the EASL/AASLD recommendations? An observational study of the HCC east-west study group. Ann Surg. 2013;257(5):929-937. https://doi.org/ 10.1097/SLA.0b013e31828329b8.

10. Abbasoglu $\mathrm{O}$. Role of liver resection in the management of multinodular hepatocellular carcinoma. World J Hepatol. 2015;7(20):2237-2240. https:// doi.org/10.4254/wjh.v7.i20.2237.

11. Kim H, Ahn SW, Hong SK, et al. Survival benefit of liver resection for Barcelona Clinic Liver Cancer stage B hepatocellular carcinoma. Br J Surg. 2017;104(8): 1045-1052. https://doi.org/10.1002/bjs.10541.

12. Zaydfudim VM, Vachharajani N, Klintmalm GB, et al. Liver resection and transplantation for patients with hepatocellular carcinoma beyond milan criteria. In: Annals of Surgery. vol. 264. Lippincott Williams and Wilkins; 2016:650-658. https://doi.org/10.1097/SLA.0000000000001866.

13. Colombo M, Sangiovanni A. Treatment of hepatocellular carcinoma: beyond international guidelines. Liver Int. 2015;35(s1):129-138. https://doi.org/ 10.1111/liv.12713.

14. Zhong JH, Ke Y, Gong WF, et al. Hepatic resection associated with good survival for selected patients with intermediate and advanced-stage hepatocellular carcinoma. Ann Surg. 2014;260(2):329-340. https://doi.org/10.1097/ SLA.0000000000000236.

15. Kokudo N, Makuuchi M. Evidence-based clinical practice guidelines for hepatocellular carcinoma in Japan: the J-HCC guidelines. I Gastroenterol. 2009;44(SUPPL. 19):119-121. https://doi.org/10.1007/s00535-008-2244-z.

16. Kudo M, Arizumi T, Ueshima K, Sakurai T, Kitano M, Nishida N. Subclassification of BCLC B stage hepatocellular carcinoma and treatment strategies: proposal of modified bolondi's subclassification (Kinki criteria). Dig Dis. 2015;33(6): 751-758. https://doi.org/10.1159/000439290.

17. Mazzaferro V, Llovet JM, Miceli R, et al. Predicting survival after liver transplantation in patients with hepatocellular carcinoma beyond the Milan criteria: a retrospective, exploratory analysis. Lancet Oncol. 2009;10(1):35-43. https:// doi.org/10.1016/S1470-2045(08)70284-5.

18. Johnson PJ, Berhane S, Kagebayashi C, et al. Assessment of liver function in patients with hepatocellular carcinoma: a new evidence-based approach - the albi grade. J Clin Oncol. 2015;33(6):550-558. https://doi.org/10.1200/ JCO.2014.57.9151.

19. Clavien PA, Barkun J, De Oliveira ML, et al. The clavien-dindo classification of surgical complications: five-year experience. Ann Surg. 2009;250(2):187-196. https://doi.org/10.1097/SLA.0b013e3181b13ca2.

20. Pecorelli A, Lenzi B, Gramenzi A, et al. Curative therapies are superior to standard of care (transarterial chemoembolization) for intermediate stage hepatocellular carcinoma. Liver Int. 2017;37(3):423-433. https://doi.org/ 10.1111/liv.13242.

21. Ciria R, López-Cillero $P$, Gallardo $A B$, et al. Optimizing the management of patients with BCLC stage-B hepatocellular carcinoma: modern surgical resection as a feasible alternative to transarterial chemoembolization. Eur J Surg Oncol. 2015;41(9):1153-1161. https://doi.org/10.1016/j.ejso.2015.05.023.

22. Zhong JH, Peng NF, You XM, Ma L, Li LQ. Hepatic resection is superior to transarterial chemoembolization for treating intermediate-stage hepatocellular carcinoma. Liver Int. 2017;37(7):1083-1084. https://doi.org/10.1111/ liv.13290.

23. Yin L, Li H, Li AJ, et al. Partial hepatectomy vs. transcatheter arterial chemoembolization for resectable multiple hepatocellular carcinoma beyond Milan criteria: a RCT. J Hepatol. 2014;61(1):82-88. https://doi.org/10.1016/ j.jhep.2014.03.012.

24. Ishizawa T, Hasegawa K, Aoki T, et al. Neither multiple tumors nor portal hypertension are surgical contraindications for hepatocellular carcinoma. Gastroenterology. 2008;134(7):1908-1916. https://doi.org/10.1053/ j.gastro.2008.02.091.

25. Yang X Di, Pan LH, Wang L, et al. Systematic review of single large and/or multinodular hepatocellular carcinoma: surgical resection improves survival. Asian Pac J Cancer Prev APJCP. 2015;16(13):5541-5547. https://doi.org/ 10.7314/APJCP.2015.16.13.5541.

26. Choi SH, Choi GH, Kim SU, et al. Role of surgical resection for multiple hepatocellular carcinomas. World J Gastroenterol. 2013;19(3):366-374. https:// doi.org/10.3748/wjg.v19.i3.366.

27. Galle PR, Tovoli F, Foerster F, Wörns MA, Cucchetti A, Bolondi L. The treatment of intermediate stage tumours beyond TACE: from surgery to systemic therapy. J Hepatol. 2017;67(1):173-183. https://doi.org/10.1016/j.jhep.2017.03.007.

28. Liu W, Wang K, Bao Q, Sun Y, Xing BC. Hepatic resection provided long-term survival for patients with intermediate and advanced-stage resectable hepatocellular carcinoma. World J Surg Oncol. 2016;14(1). https://doi.org/10.1186/ s12957-016-0811-y.

29. Zhong JH, Rodríguez AC, Ke Y, Wang YY, Wang L, Li LQ. Hepatic resection as a safe and effective treatment for hepatocellular carcinoma involving a single large tumor, multiple tumors, or macrovascular invasion. Med (United States). 2015;94(3):e396. https://doi.org/10.1097/MD.0000000000000396.

30. Tada T, Kumada $T$, Toyoda $H$, et al. Role of hepatic resection in patients with intermediate-stage hepatocellular carcinoma: a multicenter study from Japan. Canc Sci. 2017;108(7):1414-1420. https://doi.org/10.1111/cas.13257. 